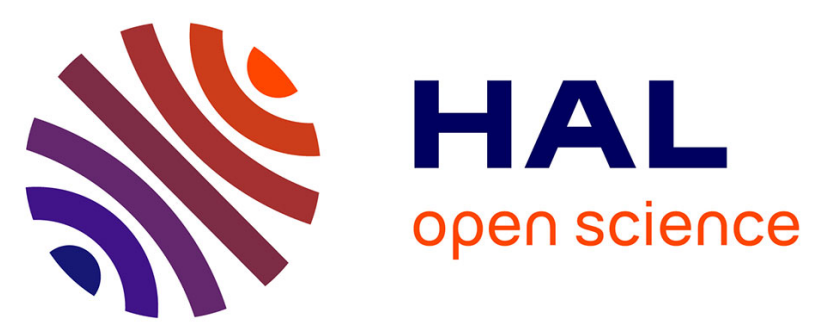

\title{
Alternating Direction Method of Multipliers Applied to 3D Light Sheet Fluorescence Microscopy Image Deblurring Using GPU Hardware
}

François de Vieilleville, Pierre Weiss, Valérie Lobjois, Denis Kouamé

\section{- To cite this version:}

François de Vieilleville, Pierre Weiss, Valérie Lobjois, Denis Kouamé. Alternating Direction Method of Multipliers Applied to 3D Light Sheet Fluorescence Microscopy Image Deblurring Using GPU Hardware. 2011 33rd Annual International Conference of the IEEE Engineering in Medicine and Biology Society (EMBC 2011), Aug 2011, Boston, France. pp.4872-4875, 10.1109/IEMBS.2011.6091207. hal-03101422

\section{HAL Id: hal-03101422 \\ https://hal.inria.fr/hal-03101422}

Submitted on 7 Jan 2021

HAL is a multi-disciplinary open access archive for the deposit and dissemination of scientific research documents, whether they are published or not. The documents may come from teaching and research institutions in France or abroad, or from public or private research centers.
L'archive ouverte pluridisciplinaire HAL, est destinée au dépôt et à la diffusion de documents scientifiques de niveau recherche, publiés ou non, émanant des établissements d'enseignement et de recherche français ou étrangers, des laboratoires publics ou privés. 


\title{
Alternating Direction Method of Multipliers Applied to 3D Light Sheet Fluorescence Microscopy Image Deblurring Using GPU Hardware
}

\author{
F. de Vieilleville, P. Weiss, V. Lobjois and D. Kouamé
}

\begin{abstract}
This paper focuses on the deblurring and denoising of Poisson noise contaminated images acquired with a new imaging technique producing large 3D data sets: Light Sheet Fluorescence Microscopy. This paper details the optimization algorithm used, which is based on the Alternating Direction Method of Multipliers, and its efficient implementation using GPU hardware. In practice, a 3D 100 million voxel image is deconvolved in five minutes, which is at least 25 times faster than a state-of-the-art MATLAB implementation.
\end{abstract}

\section{INTRODUCTION}

Light Sheet Fluorescence Microscopy (LSFM) is a recent and very promising imaging technique in which samples are illuminated with a thin light sheet instead of being globally illuminated. This approach defines a thinner optical section thereby reducing the photobleaching and phototoxicity effects, see [1]. 3D images are obtained by stacking the acquired slices while the light sheet goes through the sample which is commonly imaged under several angles of view. Depending on the set-up, a perfect alignment of the light sheet with the focal plane of the imaging objective may be hard to obtain. Then, small fluorescent beads $(\approx 0.17 \mu \mathrm{m}$ diameter $)$ embedded in agarose gel allow a good calibration of the microscope simply by looking at the obtained Point Spread Function (PSF) on the images. Consequently, the blur induced by the optic elements can be considered as space-invariant on reasonable size parts of images.

However this technology is still subjected to common limitations in fluorescence microscopy imaging due to low intensity CCD-image acquisitions and optic elements. As a result, the noise in the acquired images is driven by Poisson statistics. Moreover the practical restoration of LSFM images is highly limited by the amount of data to process since its is common to produce a 100 million voxel image for only one angle of view of the specimen. As far as we know, the restoration of LSFM images has been investigated only in few works; see [2] for some deblurring approach using several views at the same time and [3] for

This work was supported by the InNaBioSanté fundation

F. de Vieilleville and D. Kouamé are with the Université de Toulouse, IRIT UMR CNRS 5505, France \{devieill, kouame\}@irit.fr

P. Weiss is with the Université de Toulouse, IMT UMR CNRS 5219, France pierre.armand.weiss@gmail.com

V. Lobjois is with the ITAV, UMS 3039 CRT-RIV, France valerie.lobjois@itav-recherche.fr so-called fusion algorithms, sometimes with fluorescent beads [4], [5].

The simplest deconvolution methods are the Wiener and Wiener-Tikhonov linear filters which are very noisesensitive and unable to improve the restoration for zero response frequencies of the PSF [6]. The most classic iterative algorithm for Poisson statistics images is the Richardson-Lucy [7]. Other standard iterative methods for fluorescent microscopy imagery are the constrained Tikhonov-Miller or the Carrington algorithms [8] which generally assume gaussian statistics on both image priors and noise. Poisson statistics should be closer to the statistics of the acquired images in low-intensity fluorescent microscopy though this generally yields harder functional to minimize efficiently (e.g. the gradient of the Richardson-Lucy functional is not Lipchitz continuous). Thus a regularizing energy term should be introduced via maximum a posteriori (MAP) formalism.

Usually these regularizations are $\mathcal{L}^{2}$ based and yield smooth solutions, though depending on the biological marker. More fitted solutions in fluorescent image processing should be piece wise regular. The promotion of such solutions can be achieved using the TV seminorm [9], introduced in [10] for denoising purposes and in restoration of confocal microscopy images with a Richardson-Lucy based algorithm [11]. Recent litterature reports efficient methods for general $2 \mathrm{D}$ restoration of Poissonian images [12], [13] with the TV semi-norm based on the Alternating Direction Method of Multipliers (ADMM).

In this paper, we chose to promote piece wise regular solutions with Poisson statistics for 3D LSFM images. To account for the data size, we applied a fast numerical scheme implemented using GPU hardware. The numerical scheme chosen is based on the ADMM and its implementation allows us to process a 3D 100 million voxel image in less than 5 minutes with a recent hardware such as a NVIDIA CUDA device with a compute capability 1.3 or above.

The paper is organized as follows: Section II introduces the image formation model, our energy minimizing problem and the algorithm used to solve it. Section III presents the various experiments and their results. Eventually, we draw some conclusions in Section IV. 


\section{Optimization Method and Implantation}

\section{A. Image Formation Model}

We assume here that our image relies on a standard formation process with Poisson noise, i.e. :

$$
m=\mathcal{P}(h \otimes x)
$$

where $m$ is the observed image, $h$ the PSF of the optical system, $\otimes$ the convolution operator, $\mathcal{P}(\cdot)$ a process with Poisson statistics, $x$ the unknown object we wish to reconstruct and $\hat{x}$ our estimation of $x$.

Evaluating $\hat{x}$ is often conducted through the discrete Bayes' condition formula:

$$
p(a \mid b)=\frac{p(b \mid a) p(a)}{p(b)} .
$$

A first approximation to $p(x \mid m)$ is to only consider the likelihood term, that is setting $p(x)$ to one. In this case, the deblurring problem is an optimization problem where:

$$
\begin{aligned}
\hat{x} & =\underset{x \in \mathbb{R}^{n}}{\operatorname{argmin}}-\log p(m \mid x) \quad \text { and } \\
p(m \mid x) & =\prod_{i} \frac{\exp (-(h \otimes x)(i))((h \otimes x)(i))^{m(i)}}{m(i) !} .
\end{aligned}
$$

Solving this problem by a multiplicative formula is referred to as the Richardson-Lucy algorithm [7]. One step further is to consider the maximum a posteriori (MAP) formulation, in which one adds hypothesis on the unknown $x$ using priors, that is defining $p(x)$. Such approaches have been widely used in deblurring fluorescence microscopy images and several combinations have already been tried, e.g. Poisson noise with gaussian prior on $x$, Poisson prior on $x$, Gaussian noise and Gaussian prior on $x$ [14]. In this work, the nature of our LSFM images leads us to investigate an exponential-distribution type with Total Variation (TV) prior on the unknown image.

$$
p(x) \propto \prod_{\text {voxels }} \exp (-\alpha T V(x))
$$

Thus, our deblurring task is an unconstrained optimization problem expressed as:

$$
\hat{x}=\underset{x \in \mathbb{R}^{n}}{\operatorname{argmin}} h \otimes x-m \log (h \otimes x)+\alpha T V(x)
$$

\section{B. General Alternating Direction Method of Multipliers Framework}

Many numerical schemes exist to solve the previous problem, amongst the most simple and popular is the conjugate gradient algorithm. However such methods are known to be slow to converge, especially when the functional is not quadratic, non-smooth or when its gradient is not Lipchitz differentiable. Moreover the implantation of such algorithms requires a lot of work to be efficiently parallelized on GPU. In contrast, here, we fit our problem in the general framework of the ADMM (see [13]) which will appear to be perfectly fitted for GPU implantation. Let us consider $x \in \mathbb{R}^{n}, y \in \mathbb{R}^{m}, B$ a matrix with $l$ rows and $n$ columns, $C$ a matrix with $l$ row and $m$ columns and $b \in \mathbb{R}^{l}$.

$$
\underset{\text { s.t. } B x+C y=b, x \in X, y \in Y}{\operatorname{argmin}} f_{1}(x)+f_{2}(y)
$$

To solve such a problem, we denote $\mathcal{L}(x, y, \lambda)$ to be its augmented Lagrangian and the ADMM iterative algorithm reads:

- $x_{k+1}=\operatorname{argmin}_{x \in \mathbb{R}^{n}} \mathcal{L}\left(x, y_{k}, \lambda_{k}\right)$,

- $y_{k+1}=\operatorname{argmin}_{y \in \mathbb{R}^{m}} \mathcal{L}\left(x_{k+1}, y, \lambda_{k}\right)$,

- $\lambda_{k+1}=\lambda_{k}+\beta(B x+C y-b)$,

until stopping criteria are met.

\section{Problem Formulation And Explicit Algorithm}

In our specific problem $x$ denotes the 3D image which is considered as a vector of size $n$. Denoting $\langle$,$\rangle the usual$ scalar product and $H x$ the convolution of $h$ by $x$, our deblurring task (Eq. 2) fits ADMM by choosing:

- $f_{1}(x)=0, x \in \mathbb{R}^{n}$,

- $y=(w, z) \in \mathbb{R}^{n} \times \mathbb{R}^{3 n}$,

- $f_{2}(y)=\langle 1, w\rangle-\langle m, \log w\rangle+\alpha\|z\|_{1}$,

- $B=\left(\begin{array}{c}H \\ \nabla\end{array}\right), C=I d_{4 n}$ and $b=0$.

We recall that as $x$ is a $3 \mathrm{D}$ image, $\nabla(x)$ lies in $\mathbb{R}^{3 n}$. The augmented Lagrangian reads :

$$
\begin{aligned}
\mathcal{L}(x, y, \lambda)= & <1, w>-<y, \log (w)>+\alpha\|z\|_{1}+ \\
& <\lambda, B x-C y>+\frac{\beta}{2}\|B x-C y\|_{2}^{2},
\end{aligned}
$$

with $\lambda=\left(\lambda_{1}, \lambda_{2}, \lambda_{3}, \lambda_{4}\right) \in \mathbb{R}^{4 n}$. In our problem, the three-fold ADMM algorithm is decomposed as follows:

a) Step 1:

$x_{k+1}=\underset{x \in \mathbb{R}^{n}}{\operatorname{argmin}} \mathcal{L}\left(x, y_{k}, \lambda_{k}\right)=\underset{x \in R^{n}}{\operatorname{argmin}} \frac{\beta}{2}\left\|B x-C y_{k}+\frac{\lambda_{k}}{\beta}\right\|_{2}^{2}$

We choose to use circular boundary conditions which entail that $H, \nabla_{x}, \nabla_{y}$ and $\nabla_{z}$ are block-circulant matrices and that $x_{k+1}$ can be easily retrieved using Fast Fourier Transform :

$x_{k+1}=\mathcal{F}^{-1}\left(\frac{\mathcal{F}\left(H^{T}\left(w_{k}-\frac{\lambda_{1, k}}{\beta}\right)+\nabla^{T}\left(z_{k}-\frac{\left(\lambda_{2, k}, \lambda_{3, k}, \lambda_{4, k}\right)}{\beta}\right)\right)}{\mathcal{F}\left(H^{T} H+\nabla^{T} \nabla\right)}\right)$.

Note that the operations are done point-wise in the spectral domain.

b) Step 2:

$$
\begin{aligned}
& y_{k+1}=\underset{y \in \mathbb{R}^{4 n}}{\operatorname{argmin}} \mathcal{L}\left(x_{k+1}, y, \lambda_{k}\right) \\
& y_{k+1}=\underset{w \in \mathbb{R}^{n}, z \in \mathbb{R}^{3 n}}{\operatorname{argmin}}<1, w>-<m, \log (w)>+\alpha\|z\|_{1} \\
&+\frac{\beta}{2}\left\|B x_{k+1}-\left(\begin{array}{c}
w \\
z
\end{array}\right)+\frac{\lambda_{k}}{\beta}\right\|_{2}^{2}
\end{aligned}
$$


Which can be split into:

$$
\begin{aligned}
& w_{k+1}=\underset{w \in \mathbb{R}^{n}}{\operatorname{argmin}}\langle 1, w>-<m, \log (w)> \\
&+\frac{\beta}{2}\left\|w-H x_{k+1}-\frac{\lambda_{1, k}}{\beta}\right\|_{2}^{2}, \\
& z_{k+1}=\underset{z \in \mathbb{R}^{3 n}}{\operatorname{argmin}} \alpha\|z\|_{1}+\frac{\beta}{2}\left\|z-\nabla x_{k+1}-\frac{\left(\lambda_{2, k}, \lambda_{3, k}, \lambda_{4, k}\right)}{\beta}\right\|_{2}^{2} .
\end{aligned}
$$

And solved as :

$$
\begin{aligned}
& w_{k+1}=\frac{q-\frac{1}{\beta}}{2}+\frac{1}{2} \sqrt{\left(\frac{1}{\beta}-q\right)^{2}+\frac{4 m}{\beta}} \\
& z_{k+1}=\operatorname{soft}_{\frac{\alpha}{\beta}}\left(\nabla x_{k+1}+\frac{\left(\lambda_{2, k}, \lambda_{3, k}, \lambda_{4, k}\right)}{\beta}\right) .
\end{aligned}
$$

With $q=H x_{k+1}+\frac{\lambda_{1, k}}{\beta}$ and $\operatorname{soft}_{\gamma}(\cdot)$ being the soft thresholding operator :

$$
\operatorname{soft}_{\gamma}(u) \equiv \frac{u}{\|u\|_{1}} \times \max \left(\|u\|_{1}-\gamma, 0\right) .
$$

Where the operations are done point-wise with the convention that for $u(i)=(0,0,0)$, we set $\frac{u(i)}{\|u(i)\|}=$ $(0,0,0)$.

$$
\text { c) Step 3: }
$$

$$
\lambda_{k+1}=\lambda_{k}+\beta(B x-C y)
$$

Finally, our algorithm is similar to the PIDSplit algorithm of [13], except that we work on 3D images instead of $2 \mathrm{D}$ images, that the linear system is inverted in the spectral domain with a Fast Fourier Transform instead of a DCT-II and that we used preconditioners (not explicited here for simplicity).

\section{Detailed Implementation}

The previous algorithm was implemented using $\mathrm{C}++$ and CUDA, and part of the input/output routines (e.g. import/export ".tif" images) depends on a third party library. As many operations are done point-wise in the complex domain, their implantation using the Single Instruction Multiple Data paradigm in CUDA is straightforward. When all variables of the problem are considered plus some extra space used for minimizing data transfer, the program requires approximately 18 copies of the image with a complex type. Since the RAM available on a single GPU card varies generally between $500 \mathrm{Mb}$ (e.g. Quadro FX 2700M) and 4.5 Gb (e.g. Tesla C1060), the maximal image size is thus limited. As an example, a $128^{3}$ voxel image requires approximately $301 \mathrm{Mb}$ of RAM to be processed. Consequently, our strategy to process large images (e.g. between 20 and 100 million-voxel images) is to split them with a determined overlapping ratio varying with the support function of the blur involved in the image formation process. This is possible because the energy of the PSF is spatially concentrated. To maintain a constant execution time for a given size of image, the stopping criterion chosen is a fixed number of iterations. This iteration number was chosen so that the intensity variation of each voxel can be considered visually neglectible for further iterations. Moreover, after 50 iterations, the energy on our image sets, it diminished of a factor $10^{3}$ from an approximation of the solution of the problem, that is $\left|E\left(x_{50}\right)-E\left(x_{N}\right)\right|<10^{-3} \mid E\left(x_{1}\right)-$ $E\left(x_{N}\right) \mid$ with $N$ very large.

\section{E. Point Spread Function}

A very important input parameter of the algorithm is the PSF. To estimate it in our experiments, we average the response of some fluorescent beads embedded in agarose gel around the sample. The chosen responses have a pixel intensity sum laying within a bound determined by a confidence interval.

\section{Experiments, Results And Discussion}

The experiments were conducted on two sets of images, using three different GPU cards, with the same block size. The first set is an open set of LSFM images 1 representing a relatively transparent biological sample (Drosophila embryo expressing His-YFP) for which our image formation model can be considered as valid. As shown by the results, in Fig. 1, the deconvolution effect is clear and yields a much better resolution of the sample.

The second set of images was acquired with an experimental set-up illustrated on Fig. 2 and represents Multi Cellular Tumor Spheroids $(\approx 500 \mu \mathrm{m}$ diameter spheres), marked with propidium iodide (nuclei), see Fig. 3. Although high scattering effects are observed as a smooth variant positive background value, the impact of the algorithm is clearly visible, making some biological phenomena, such as DNA concentration during the proliferation phase, much more quantifiable. Regarding the execution time, recent GPU hardware behaves faster due to a higher number of cores but also due to more efficient memory transfers, see Table I. Even if it is difficult to compare, the authors of [13] report a computation time of 158 seconds on a two $2.4 \mathrm{Ghz}$ processor desktop for a 2D 256x256 pixel image. Assuming a processing time linearly dependant of both the number of iterations and the number of points, which is an underestimation since part of the algorithm runs with a $\mathcal{O}(n \log (n))$, the projected required time for the Drosophila image would be around 2964.4 seconds with 50 iterations. Compared to our results, we see that the CUDA implementation of our algorithm brings the computation time down by a factor of 25 to 50 for recent CUDA compatible devices.

\section{Conclusion}

In this paper we have proposed an implementation on GPU device of an ADMM based algorithm for the restoration of blurred 3D images contaminated by Poisson noise. The obtained processing time are compatible with a routine exploitation on LSFM images.

\footnotetext{
${ }^{1}$ See http://pacific.mpi-cbg.de/wiki/index.php/SPIM_ Registration_Method and [5]
} 

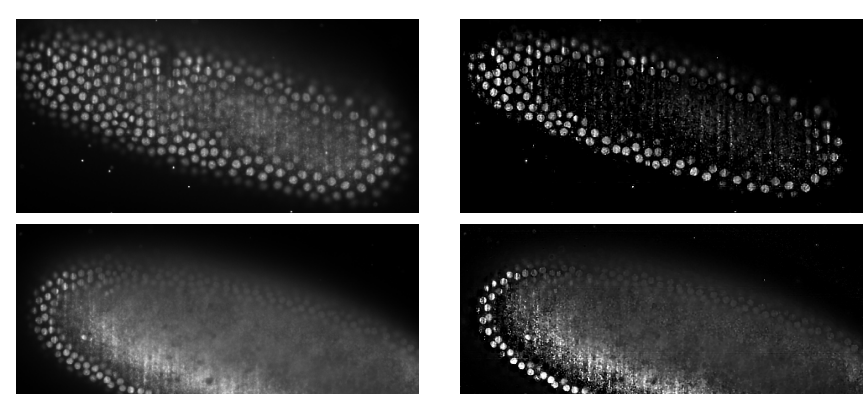

Fig. 1. Results using a biological marker on a drosophila transparent sample. (Left) Partial slices of an acquired 3D image drosophila embryo expressing His-YFP. (Right) Corresponding deconvolved slice using our algorithm, $\alpha=0.002, \beta=0.002,50$ iterations.

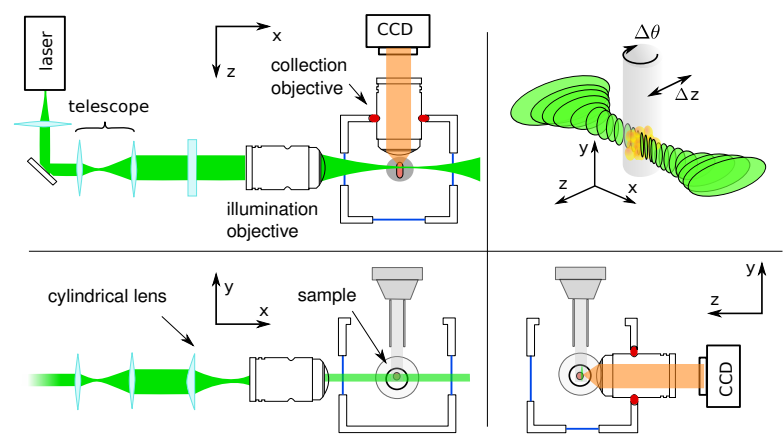

Fig. 2. Light sheet fluorescence microscopy set-up used for propidium iodide marked spheroid acquisitions.
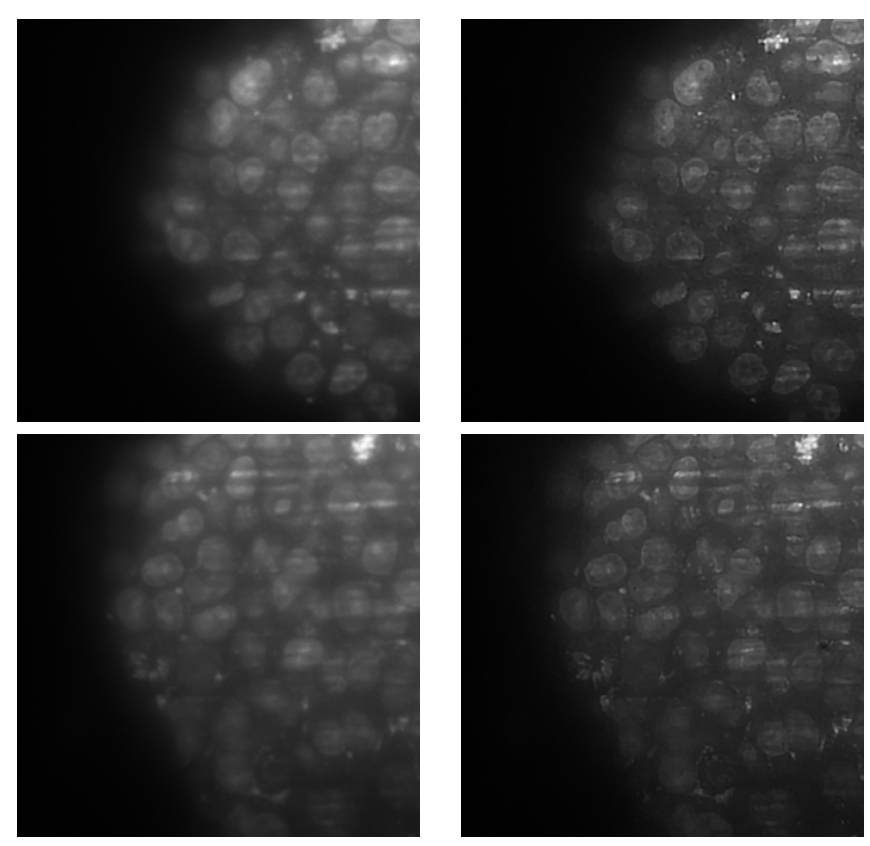

Fig. 3. Results using a biological marker with high scattering effects. (Left) Partial slices of an acquired 3D image with our set-up using IP marker on fixed MCTS $500 \mu \mathrm{m}$ diameter. (Right) Corresponding deconvolved slice using our algorithm, $\alpha=0.002$, $\beta=0.002,50$ iterations.
TABLE I

Computing times For the tWo TeSt SETS OF IMAGES USED , DEPENDING ON THE HARDWARE USED.

$953 \times 543 \times 81$ voxels drosophila embryo 3D image.

\begin{tabular}{|c|c|c|}
\cline { 2 - 3 } \multicolumn{1}{c|}{} & Cores & Process time (s) \\
\hline FX2700M & 48 & 544,729 \\
\hline TESLA C1060 & 250 & 113,25 \\
\hline GTX 580 & 500 & 61,300 \\
\hline
\end{tabular}

$338 \times 338 \times 200$ voxels multi-cellular tumor spheroid 3D image.

\begin{tabular}{|c|c|c|}
\cline { 2 - 3 } \multicolumn{1}{c|}{} & Cores & Process time (s) \\
\hline FX2700M & 48 & 398,72 \\
\hline TESLA C1060 & 250 & 73,85 \\
\hline GTX 580 & 500 & 38,106 \\
\hline
\end{tabular}

\section{REFERENCES}

[1] J. Huisken and D. Stainier, "Selective plane illumination microscopy techniques in developmental biology," Development (Cambridge, England), vol. 136, pp. 1963-75, June 2009.

[2] P. Verveer, J. Swoger, F. Pampaloni, K. Greger, M. Marcello, and E. Stelzer, "High-resolution three-dimensional imaging of large specimens with light sheet-based microscopy," Nature Methods, vol. 4, pp. 307-8, April 2007.

[3] S. Preibisch, T. Rohlfing, M. P. Hasak, and P. Tomancak, "Mosaicing of single plane illumination microscopy images using groupwise registration and fast content-based image fusion," in proc. of SPIE, Medical Imaging Symposium : Image Processing, vol. 6914, 2008.

[4] S. Preibisch, S. Saafeld, T. Rohlfing, and P. Tomancak, "Bead-based mosaicing of single plane illumination microscopy images using geometric locla descriptor matching," Progress in biomedical optics and imaging, vol. 10, no. 35, 2009.

[5] S. Preibisch, S. Saalfeld, J. Schindelin, and P. Tomancak, "Software for bead-based registration of selective plane illumination microscopy data," Nature Methods, vol. 7, no. 6, pp. 418-419, 2010.

[6] H. van der Voort and K. Strasers, "Restoration of confocal images for quantitative image analysis," Journal of Microscopy, vol. 178, pp. 165-181, 1995.

[7] L. Lucy, "An iterative technique for the rectification of observed distributions," The astronomical journal, vol. 79, no. 6, June 1974.

[8] G. van Kempen, L. van Vliet, P. Verveer, and H. van der Voort, "A quantitative comparison of images restoration methods for confocal microscopy," Journal of Microscopy, vol. 185, pp. 354-365, 1996.

[9] R. Acar and C. Vogel, "Analysis of bounded variation penalty methods for ill-posed problems," Inverse Problems, vol. 10, pp. 1217-1229, 1994.

[10] L. Rudin, S. Osher, and E. Fatemi, "Nonlinear total variation based noise removal algorithms," Physica D, no. 60, pp. 259$268,1992$.

[11] N. Dey, L. Blanc-Feraud, C. Zimmer, P. Roux, Z. Kam, J.C. Olivo-Marin, and J. Zerubia, "Richardson-lucy algorithm with total variation regularization for $3 \mathrm{~d}$ confocal microscope deconvolution," Microscopy Reasearch and Technique, vol. 69, pp. 260-266, 2006.

[12] M. A. T. Figueiredo and J. M. Bioucas-Dias, "Restoration of Poissonian Images Using Alternating Direction Optimization," IEEE Transactions on Image Processing, vol. 19, no. 12, pp. 3133-3145, Dec. 2010.

[13] S. Setzer, G. Steidl, and T. Teuber, "Deblurring poissonian images by split bregman techniques," Journal of Visual Communication and Image Representation, vol. 21, pp. 193199, 2010.

[14] P. Verveer and T. Jovin, "Efficient superresolution restoration algorithms using maximum a posteriori estimations with application to fluorescence microscopy," Journal of Optical Society of America, vol. 14, no. 8, August 1997. 